\title{
Reducing ionizing radiation in children and adolescents
}

Accurate staging of tumours in children and adolescents is of utmost importance, but PET-CT staging is associated with substantial exposure to ionizing radiation. Moreover, the diagnostic tests that a child has to undergo can be stressful, time consuming, expensive and may require repetitive anaesthesias. This situation prompted Heike Daldrup-Link and colleagues to "develop a single 'one-stop shop' imaging test, which could provide a comprehensive evaluation of the primary tumour and metastases in one session, but not be associated with any radiation exposure."

The researchers used MRI, which is based on magnetic fields rather than radiotracers or X-rays. Using a combination of anatomical and functional imaging, they established a novel whole-body diffusion-weighted MRI approach that uses iron oxide nanoparticles to improve tumour delineation. The researchers first acquired scans that defined very accurately the anatomy of the patients and then obtained scans that depicted tumours as bright spots with little or no background. They used an iron supplement as a contrast agent, "the iron supplement can be detected by the MRI magnet and was able to improve tumour detection and vessel delineation," explains Daldrup-Link. She continues, "we then fused the anatomical scans with the tumour scans, to produce integrated whole-body diffusion-weighted scans that look like PET-CT scans, but are not associated with any radiation exposure."

The results of this new approach were impressive: the radiation free whole-body diffusion-weighted MRI technique detected 158 of 174 malignant tumours, which was similar to results obtained with PET-CT (163 of 174 malignant tumours). The sensitivity, specificity and diagnostic accuracy

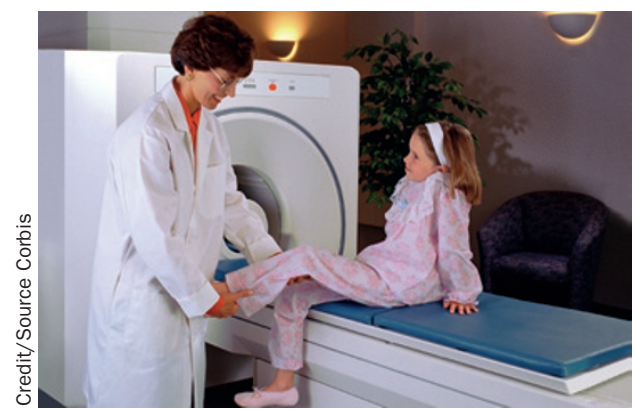

between this new approach and standard ionizing-based imaging were excellent, with values above $90 \%$. Importantly, both technologies required roughly the same time between tracer injection and scan result, and the estimated costs for both approaches were comparable. "We demonstrate that it is possible to provide sensitive tumour staging of children with malignant tumours using a completely radiation-free imaging test as an alternative to PET-CT scans," says Daldrup-Link.

The researchers note that this technique has so far only been proven for certain types of lymphomas, and that additional studies are needed to demonstrate its utility for treatment monitoring. "Nearly every primary solid tumour in children is currently evaluated with MRI, requiring at least two imaging tests: an MRI for evaluation of the primary tumour and a radiotracer scan to evaluate potential metastases. Our 'one-stop shop' whole-body staging exam that can be used at the same time of evaluation of the primary tumour would be much easier for the patient, saves time and avoids the need for duplicate anaesthesias," concludes Daldrup-Link.

\section{Lisa Hutchinson}

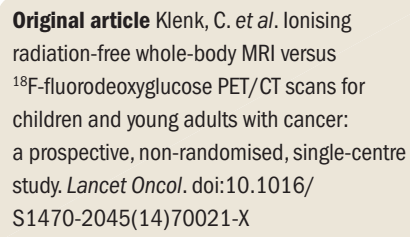

